\title{
SUPERCRITICAL DEFORMATIONS
}

c. P. Cherepanov

UDC $539.375+539.4$

1. Introduction. One of the basic characteristics of an elastoplastic material is stress-strain or load-displacement type unidimensional quasistatic curves. These, for example, include standard $\sigma-\varepsilon$ (tension-compression of long prismatic samples) and $\tau-\gamma$ (torsion of a thin-walled hollow round tube) curves. The curves of this type observed in tests have two portions; first, with an increase in strain the stress increases (subcritical portion) and then, beyond a certain critical condition at which the stress reaches a maximum, 1t decreases (supercritical portion). The relationship of the characteristic load to the characteristic displacement changes the same way for an elastoplastic body of any form.

From simple quasistatic considerations it is clear that any position on the supercritical branch is unstable. Therefore the supercritical portion is a dynamic characteristic of the specimen-test machine system in general (in contrast to the subcritical portion, which is a property only of the material, the form of the specimen, and the form of external load).

Nevertheless, it is natural to assume (as many authors have) that the quasistatic supercritical branch of the curve does exist as some characteristic of the material itself with corresponding equations of the elastoplastic condition (as in the subcritical area). Then there arises the specific elastoplastic problem with zones of the subcritical and supercritical plastic condition subject to determination.

2. $\sigma-\varepsilon$ and $\tau-\gamma$ Curves. Let us first consider unidimensional cases of simple tension of a rod and simple shear of a layer. In tension, for simplicity, let us assume that the deformation in the whole rod is small so that the reduction in area of the rod in the supercritical area (neck) is small, that is, only the initial supercritical portion adjoining the critical portion is considered. With such an assumption the case of simple tension of a rod will be mathematically identical to the case of simple shear of a layer and therefore we will limit ourselves to the first case.

The $\sigma-\varepsilon$ curve of an elastoplastic material is described by the following rule:

$$
\dot{\sigma}=\left\{\begin{array}{c}
\sigma^{\prime}(\varepsilon) \dot{\varepsilon} \text { with } \dot{\varepsilon}>0 \text { (loading); } \\
\dot{E} \dot{\varepsilon} \text { with } \dot{\varepsilon}<0 \text { (unloading). }
\end{array}\right.
$$

where $\dot{\sigma}=\partial \sigma / \partial \mathrm{t} ; \dot{\varepsilon}=\partial \varepsilon / \partial \mathrm{t} ; \sigma^{\prime}(\varepsilon)=\mathrm{d} \sigma / \mathrm{d} \varepsilon$.

For specificness let us consider supercritical deformation in the small vicinity of the critical condition, which we take as the point from which the stresses $\sigma$, the strains $\varepsilon$, and the displacements $u$ are measured (Fig. 1). Therefore, we consider the small disturbances close to the initial undisturbed condition in which the rod is subjected to the stresses $\sigma_{t}$ of uniform strain $\varepsilon_{t}$ (Fig. 1).

We should note that, as is easy to verify, all of the general results obtained below for the critical initial condition are valid for the supercritical initial conditions.

Let us introduce the designations: $t$ - time; $x$ - the coordinate along the axis of the rod with the origin at the center of the rod; $2 l$ - the length of the rod; $\rho$ and $E$ - the density and Young's modulus, respectively; $\sigma(\varepsilon)$ - the relationship of $\sigma$ to $\varepsilon$ in simple loadIng (Fig. 1).

The evolutionary equation of the theory of small disturbances in the given case is

$$
\theta \frac{\partial^{2} u}{\partial x^{2}}=\frac{\partial^{2} u}{\partial t^{2}}
$$

All-Union Scientiffc-Research Institute for Drilling Technology, Ministry of the Petroleum Industry, Moscow. Translated from Problemy Prochnosti, No. 8, pp. 3-8, August, 1985. Original article submitted Apri1 9, 1984. 


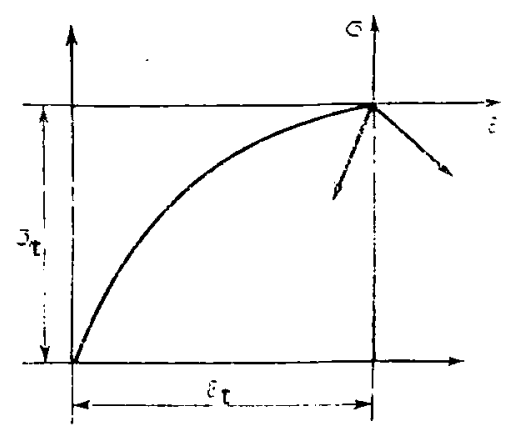

Fig. 1. The $\sigma-\varepsilon$ curve close to the critical condition.

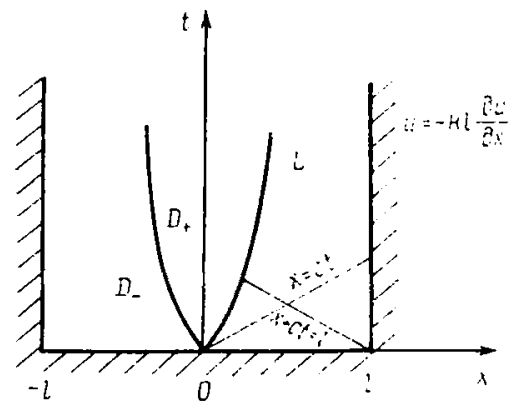

F1g. 2. The area of representation of the boundary problem on the xt plane.

where

$$
\theta=\left\{\begin{aligned}
&-a^{2} \text { with } \frac{\partial u}{\partial x}>0, \quad \frac{\partial^{y} u}{\partial x \partial t}>0 \text { (loading); } \\
& c^{2} \text { with } \frac{\partial u}{\partial x}<0 \text { and with } \frac{\partial u}{\partial x}>0, \frac{\partial^{2} u}{\partial x \partial t}<0 \\
& \text { (unloading); }
\end{aligned}\right.
$$

Therefore in the area of loading $D_{+}$on the plane xt the Laplace equations occurs and

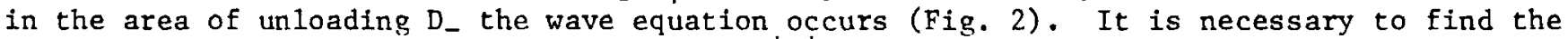
solution of Eq. (2) in the half-strip $t \geqslant 0,|x| \leqslant Z$ satisfying the following boundary and initial conditions:

with $\mathrm{x}= \pm l$

$$
u=\mp l k(t) \frac{\partial u}{\partial x}
$$

with $t=0$

$$
u=\varphi_{0}(x), \quad \frac{\partial u}{\partial t}=\Psi_{0}(x)
$$

where $\varphi_{0}(x)$ and $\psi_{0}(x)$ are certain functions (external excitations, which we will assume to be infinitely small) and $k(t)$ is the coefficient of rigidity of fastening, which we will assume to be linearly elastic (with $k \rightarrow 0$ the fastening will be absolutely rigid and with $k \rightarrow \infty$ ideally yielding), and in the general case the value of $k$ may depend upon time.

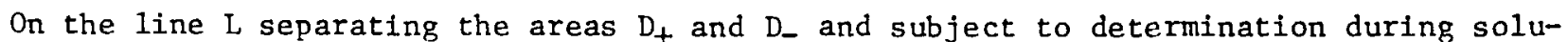
tion of the problem, the following conditions must be fulfilled

$$
\begin{gathered}
u_{+}=u_{-} \text {(the condition of continuity) } \\
c^{2}\left(\frac{\partial u}{\partial x}\right)_{-}=-a^{2}\left(\frac{\partial u}{\partial x}\right)_{+} \text {(the equation of equilibrium) }
\end{gathered}
$$

where the subscripts " + " and "-" refer to the areas $D_{+}$and $D_{-}$, respectively. 
As may be seen, the derivative $\partial u / \partial x$ has a fump at the line $L$. The area of loading $D_{+}$ and its boundary L obviously describe the evolution of the specimen neck.

Let us write simple symmetric solutions of the formulated boundary problem (2)-(6) in the two limiting cases:

the quasistatic solution (with $\partial^{2} u / \partial t^{2}=0, k=$ const)

$$
u= \begin{cases}\varepsilon_{+} x & \text { with } 0<x<x_{*}\left(D_{+}\right) ; \\ \varepsilon_{+} x_{*}+\varepsilon_{-}\left(x-x_{*}\right) & \text { with } x_{\bullet}<x<l\left(D_{-}\right)\end{cases}
$$

where

$$
\varepsilon_{+}=-\frac{c^{2}}{a^{2}} \varepsilon_{-} ; x_{*}=l \frac{a^{2}(k+1)}{c^{2}+a^{2}} ; u(-x)=u(x) ;
$$

the dynamic solution (with $0<t<\frac{1}{2} l / c, \varphi_{0}=\psi_{0}=0$ )

$$
u=\left\{\begin{array}{cl}
\varepsilon_{+} x & \text { with } 0<x<\xi t\left(D_{+}\right) \\
\varepsilon_{-}(x-c t) & \text { with } \xi t<x<c t\left(D_{-}\right) \\
0 & \text { with } 0<c t<x,
\end{array}\right.
$$

where

$$
\begin{gathered}
\varepsilon_{+}=-\frac{c^{2}}{a^{2}} \varepsilon_{-} ; \quad \xi=\frac{c a^{2}}{c^{2}+a^{2}} ; \\
u(x, t)=u(-x, t) .
\end{gathered}
$$

Here $\varepsilon_{+}$and $\varepsilon_{-}$are the uniform deformation of the rod in the area of loading and unloading, respectively $\left(\varepsilon_{+}>0, \varepsilon_{-}<0\right)$. Solutions $(7)$ and (8) contain a single free parameter, as which $\varepsilon_{-}$may be taken.

We should note that according to the quasistatic solution (7) the record of the displacement and the stress in the fastenings of the rod with $x= \pm 2$ gives the following deformation $\varepsilon_{\text {exp }}$ and stress $\sigma_{\text {exp }}$ :

$$
\begin{gathered}
\varepsilon_{\exp }=\left.\frac{1}{l} u\right|_{x=l}=-k \varepsilon_{-} ; \\
\sigma_{\exp }=E \varepsilon_{-} .
\end{gathered}
$$

Consequently, the experimentally observed $\sigma-\varepsilon$ curve in the initial supercritical portion will have the form

$$
\sigma_{\exp }=-\frac{1}{k} E \varepsilon_{\exp }
$$

that is, it is completely independent of the physical properties of the material in the supercritical area.

Now let us assume that the rigidity of fastening $k$ depends upon the time $t$. In this case there is the following alternative: the solution of the boundary problem (2)-(6) does not exist or, if tt does exist, it does not satisfy the physical principle of casuality.

The Proof. Let us assume that a solution of the boundary problem (2)-(6) does exist. In this case the solution at the arbitrary point $(x, t) \in D_{+}$, according to the general property of solutions of a Laplace equation, depends upon the boundary values of $u$ along the whole Iine $I$, that is, upon the boundary conditions along the whole boundary of the half-strip $t=0$ and $|x|=z$. Consequently, the solution of $u(x, t)$ at some arbitrary moment of time $t=t_{0}$ depends upon the rigidity of fastening $k(t)$ in the subsequent moments of $t^{i m e} t_{>} t_{0}$, which contradicts the principle of causality. Let us recall that according to the principle of causality any event occurring at the moment of time $t=t_{0}$ may depend only upon the events occurring in the preceding moments of time $t \leqslant t_{0}$.

The proven alternative is obviously also valid in that case when in the area of unloading the material is an arbitrary nonlinearly elastic one satisfying the condition $E(\varepsilon)>0$ and in the zone of loading in the supercritical area the function $\sigma(\varepsilon)$ is also arbitrary and satisfies only the condition $\sigma^{1}(E)<0\left(\mathrm{Eq}\right.$. (1)). Actually, in this case in the area $\mathrm{D}_{+}$, there will occur a quasilinear equation of elliptical type (and in the area $D_{-}$of hyperbolic type). In this case the proof is completely maintained. 


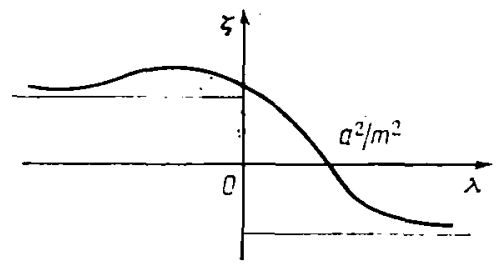

Fig. 3. The relationship of the width of the neck to the eigenvalue of the boundary problem in the supercritical area.

Failure of the principle of causality in situations considered the simplest but actually the most typical provides a basis for expecting the same in similar but more complex two- and three-dimensional elastoplastic problems. In particular, this problem has cardinal value for various theories of local failure (such as for the theory of cracks) using the concept of the supercritical strength of a material. Contradiction of this concept to the principle of causality makes it possible to conclude that in the supercritical area it is impossible to use the hypothesis of continuity and that in the material there appear discontinuities, cracks and dislocations, which must be taken into consideration explicitly.

To observe the principle of causality it is necessary that the evolutionary equations of mathematical physics not have an area of ellipticity. Therefore the zone of supercritical deformations in a correct elastoplastic model must be simulated by discontinuities, cracks, and dislocations introduced explicitly.

3. The Influence of Low Viscosity. Let the material in the supercritical condition also possess in parallel a disappearingly small $\mu \rightarrow 0$ so that the material equations of the condition may be written in the form

$$
\dot{\sigma}=\left\{\begin{array}{l}
\sigma^{\prime}(\varepsilon) \dot{\varepsilon}+\mu \ddot{\varepsilon} \text { with } \dot{\varepsilon}>0 \text { (loading) } \\
\dot{E \varepsilon}+\mu \ddot{\varepsilon} \text { with } \dot{\varepsilon}<0 \text { (unloading) }
\end{array}\right.
$$

The evolutionary equation of small disturbances in the vicinity of the critical condition in this case will be the following (designations the same as in Eqs. (2)):

$$
\theta \frac{\partial^{2} u}{\partial x^{2}}+m^{2} \frac{\partial^{3} u}{\partial x^{2} \partial t}=\frac{\partial^{2} u}{\partial t^{2}}\left(m^{2}=\frac{\mu}{\rho} \rightarrow 0\right) \text {. }
$$

This equation is of parabolic type everywhere on the plane xt (the characteristics are the straight lines $x=$ const and $t=$ const). The correct boundary problem for it will be:

$$
\begin{gathered}
\text { with } t=0 \quad u=\varphi_{0}(x) ; \quad \text { with } x= \pm l \quad u=\mp l k \frac{\partial u}{\partial x} \\
\text { at } L: u_{+}=u_{-} ; \quad c^{2}\left(\frac{\partial u}{\partial x}\right)_{-}+m^{2}\left(\frac{\partial^{2} u}{\partial x \partial t}\right)_{-}=-a^{2}\left(\frac{\partial u}{\partial x}\right)_{+}+m^{2}\left(\frac{\partial^{2} u}{\partial x \partial t}\right)_{+}
\end{gathered}
$$

The solution of boundary problem (12)-(14) with arbitrarily small values of $\mathrm{m}^{2}$ satisfies the principle of causality.

Therefore, in contrast to the elastoplastic model (2) which is incorrect in the supercritical area, our model (11) is correct. Consequently, correct formulation of the material equations of condition in the supercritical area must include taking viscosity into consideration.

Let us investigate the solution of the boundary problem (12)-(14) with $t \rightarrow \infty$. Since this problem with $t \rightarrow \infty$ assumes a group of transformations $t^{\prime}=t+C_{1}, u^{\prime} \rightarrow C_{2} u\left(C_{2}\right.$ and $C_{2}$ are the parameters of the group), its solution has the form [1]

$$
u=U(x) e^{\lambda t} \text { with } l \rightarrow \infty \text {, }
$$

where $\lambda$ is the eigenvalue of the problem and $U(x)$ is a certain function.

From this on the basis of Eqs. (12)-(14) we have

$$
\begin{gathered}
U^{\prime \prime}=\Delta^{2} U_{\text {with }} 0<x<l ; \quad U=0 \text { with } x=0 ; \quad U=-k l U^{\prime} \text { with } x=l ; \\
U_{+}=U_{-} \text {with } x=\zeta ; \quad\left(a^{2}-\lambda m^{2}\right) U_{+}^{\prime}=-\left(c^{2}+\lambda m^{2}\right) U_{-}^{\prime},
\end{gathered}
$$


where $\Delta^{2}=\frac{\lambda^{2}}{\theta+\lambda m^{2}}$.

The solution of boundary problem (16) will be

with $0<x<\xi$ :

$$
U=A \frac{\sin \left(x \Delta_{1}\right)}{\sin \left(\zeta \Delta_{1}\right)} \mathrm{e}^{\zeta \Delta_{2}}\left[1+\frac{k l \Delta_{2}+1}{k l \Delta_{2}-1} \mathrm{e}^{2(l-\zeta) \Delta_{2}} ;\right.
$$

with $\xi<x<l$ :

$$
U=A\left[\mathrm{e}^{x_{1}}+\frac{h l \Delta_{2}+1}{k l \Delta_{2}-1} \mathrm{e}^{(2 l-x) \Delta_{2}}\right]
$$

where $\Delta_{1}^{2}=\frac{\lambda^{2}}{a^{2}-\lambda m^{3}}, \Delta_{2}^{2}=\frac{\lambda^{2}}{c^{2}+\lambda m^{2}}$, and $A$ is an undetermined constant .

The number $\lambda=\lambda(\zeta)$ is determined by the following characteristic equation of the problem:

$$
\lambda\left[V^{\prime} \frac{\overline{a^{2}-\lambda m^{2}}}{c^{2}+\lambda m^{2}}-\operatorname{tg} \frac{\zeta \lambda}{\sqrt{a^{2}-\lambda m^{2}}} \operatorname{th} \frac{\lambda(\alpha+l-\zeta)}{\sqrt{c^{2}+\lambda m^{2}}}\right]=0
$$

where

$$
\exp \frac{2 \pi \lambda}{\sqrt{c^{2}+\lambda m^{2}}}=\frac{k \lambda l+\sqrt{c^{2}+\lambda m^{2}}}{k \lambda l-\sqrt{c^{2}+\lambda m^{2}}}
$$

We should note that the eigenvalue $\lambda$ may not be complex, that is, Im $\lambda=0$. In the opposite case, according to (15), with $t \rightarrow \infty$ the deformation at any fixed point will change sign, that is, the condition of loading or unloading is impossible to observe at any single point of the body.

The solution with $\lambda=0$ corresponds to the presence of a quasistatic zone of loading with $t \rightarrow \infty$. It is not difficult to see that the quasistatic solution does not depend upon the viscosity of the material and coincides with solution (7).

The coordinate $\zeta$ of the zone of loading with $t \rightarrow \infty$ is not determined directly from the solution of the boundary problem (12)-(14). To find $\zeta$, we use the global principle of the maximum of the rate of dissipation of energy proposed in [2]. According to this principle, the rate of dissipation of energy of the whole system in general is a maximum in relation to any of the free parameters of the system. From this principle, in particular, results the local principle of Mises and the associated rule of plasticity [1]. In our problem the rate of disstpation of energy of the whole rod with $\mathrm{m}^{2} \rightarrow 0$ is equal to

$$
\dot{D}=2 \sigma_{\mathrm{t}} \int_{0}^{b} \dot{\varepsilon} d x=2 \lambda \sigma_{\mathrm{t}} \mathrm{e}^{\lambda t} U(\zeta) .
$$

From this it follows that with $t \rightarrow \infty$ that value of $\zeta$ which corresponds to the maximum in $\lambda(\zeta)$ is obtained. We should note that a simllar requirement from intuitive physical considerations is advanced, for example, in the Taylor theory of hydrodynamic instability of contact phenomena [3].

With very small values of $\mathrm{m}^{2}$ the characteristic Eq. (19) acquires the following form:

$$
\frac{a}{c}-\frac{\eta-1}{\eta+1} \operatorname{tg} \frac{\lambda \zeta}{a}=\frac{\lambda m^{2}}{a c} F
$$

where

$$
\begin{gathered}
\eta=\frac{k \lambda l+c}{k \lambda l-c} \exp \left[\frac{2 \lambda}{c}(l-\zeta)\right] ; \\
F=\frac{1}{2}\left(1+\frac{a^{2}}{c^{2}}\right)+\frac{\eta-1}{\eta+1} \frac{\zeta \lambda c}{2 a^{2}} \cos ^{-2}\left(\frac{\lambda \zeta}{a}\right)+\frac{2 a \lambda \eta}{(\eta+1)^{2}} \operatorname{tg} \frac{\lambda \zeta}{a}\left(\frac{k l}{k^{2} \lambda^{2} l^{2}-c^{2}}-\frac{l-\zeta}{c^{2}}\right) .
\end{gathered}
$$

Let us write additional hypotheses normally well fulfilled in tests:

$$
\frac{a}{c} \ll 1 ; \quad \frac{k \lambda l}{c} \ll 1
$$

At the same time the characteristic Eq. (21) is transformed to simple form:

$$
\frac{\zeta}{l}=\left(\frac{a^{2}}{c^{2}} \frac{c}{\lambda l}-\frac{m^{2}}{c l}\right) \text { th } \frac{\lambda l}{c} \text {. }
$$


The $\zeta=\zeta(\lambda)$ curve constructed with the use of Eq. (23) is shown in Fig. 3. Only the positive values of $\zeta$ have a physical sense and therefore to the global principle of the maximum obviously corresponds only a single point on this curve:

$$
\zeta=0 ; \quad \lambda=\lambda_{\max }=a^{2} / m^{2} .
$$

Therefore, according to Eq. (24) for materials and test stands satisfying conditions (22), the zone of loading in the supercritical area (neck) does not propagate along the specimen, but localization of the plastic deformations in the initial cross section of origin of the neck occurs. This fact, well-known from experience, is valid for the majority of solid materlals but strangely until now has not found a satisfactory theoretical explanation.

Therefore on the basis of a correct elastoviscoplastic model it has been possible to prove that the whole zone of supercritical deformations is simulated by crack or dislocation type discontinuities of the material. This confirms the heuristic conclusion of the preceding paragraph.

It should be noted that this fundamental result is based on the global principle of the maximum [2], which has a very general thermodynamic nature similar to the Prigogine principle in irreversible thermodynamics. In the formal mathematical solution all values of $\zeta \geqslant 0$ to which correspond the numbers $\lambda$ in the range $-\infty<\lambda<a^{2} / m^{2}$ are possible. There even exist stable solutions with a finite zone of loading (neck) corresponding to negative values of $\lambda$ (Fig. 3).

In the situation composed there is no doubt of the extreme importance of meticulous experimental investigations of the $\sigma \tau \varepsilon$ and $\tau-\gamma$ curves in the near-critical area with precision measurement of the zones of loading and unloading. Such investigations make it possible to establish the boundarles of applicability of these general principles.

In conclusion we will incidentally give an answer to one interesting question: is the formation of a neck, that is, the existence of zones of loading and unloading, possible in the subcritical area? Let us turn to Fig. 1 and Eqs. (11). The analyt1cal theory presented is easily extended to the subcritical case. For this it is necessary in all equations to assume the value of $a^{2}$ as negative (that is, $a$ is imaginary). In particular, in the characteristic Eq. (23) the sign of $\alpha^{2}$ must change to the opposite. The curve of the $\zeta=\zeta(\lambda)$ relationship constructed with the use of the equation obtained is shown in Fig. 4. Nonnegative values of $\zeta$, that is, the range of $\lambda$ in which $-\infty<\lambda<-\sigma^{\prime}(\varepsilon) /\left(\rho \mathrm{m}^{2}\right)$, have a physical sense. Since all of these values of $\lambda$ are negative, the solution constructed is asymptotically stable. Therefore formation of a neck in the subcritical area is possible and in contrast to the supercritical case the neck will be stable.* As before, to the global principle of the maximum corresponds only the boundary point $\zeta=0$, that is, the width of the zone of loading with $t \rightarrow \infty$ is equal to zero within the limits of the theory of small deformations. To the value of $\zeta=0$ corresponds the solution with a break in displacements at $x=0$.

4. Curves with an Upper Yield Point. Until now curves with a single peak of the type shown In Fig. 5a have been considered. More complex cases are studied similarly. Here we will be limited to the simple shear of a layer under the action of a tangential stress $\tau$, and the layer is assumed to also be subfected to compression by a pressure p (Fig. 5b).

In this case, low-carbon steel has a $\tau-\gamma$ curve with an upper yield strength of the type shown in Fig. 5c. Actually, such a curve is characteristic of all crystals containing mobile interstitial atoms in their structure. The latter are attracted by the nucleus of a dislocation and retard it [4]. (In carbon steels, carbon atoms are interstitial atoms.)

The general theory of irreversible deformations and fracture of solids with point and linear defects from positions of invariant $\Gamma$-integrals was presented in [4]. In the continual variation it leads to a closed system of differential equations of the "theory of elasticity with three-dimensional forces and with linear features plus the theory of diffusion-drift of point defects". Curves of the type shown in Fig. 5a, c, d may, in principle, be calculated from these equations. Within the limits of the phenomenological approach used here the results of the preceding sections 2 and 3 in the given case make it possible to draw the following conclusion: the plastic area first propagates in the form of a dislocation or a slip

\footnotetext{
*In practice a neck is formed even in the subcritical area, which is the result of the presence in the system of finite disturbances, the role of which is played by structural imperfections of the grain boundary and microcracks and also by residual microstresses of a technological nature.
} 


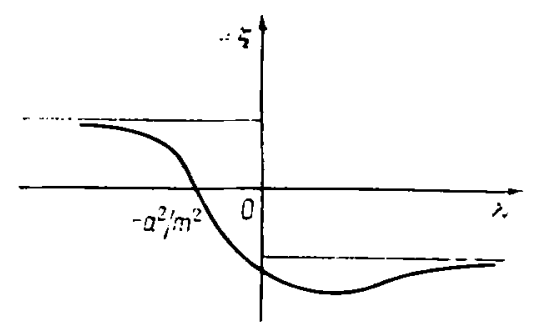

Fig. 4. The relationship of the width of the neck to the eigenvalue of the boundary problem in the subcritical area.
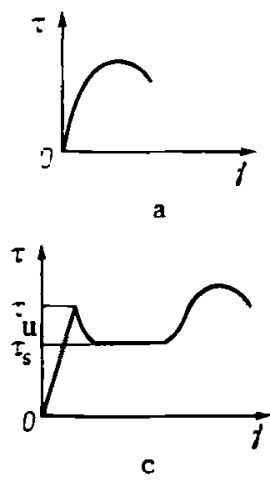

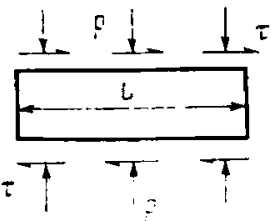

b

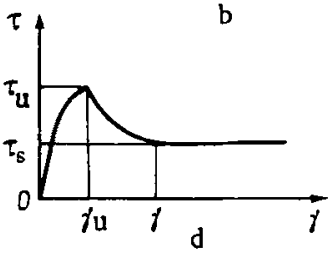

Fig. 5. Simple shear of a layer under the action of a tangential stress: a) the $\tau-\gamma$ curve considered in sections $1-3$; b) plan of simple shear; c) the $\tau-\gamma$ curve for low-carbon steel type materials; d) the $\tau \rightarrow \gamma$ curve for geological materials.

line along the plane of cleavage $L$ (it is assumed that it is parallel to the surface of the layer), that is, In the form of a surface of break in the displacement in the surrounding elastic material. This theoretical conclusion agrees very well with experimental data. Let us recall that phenomenologically the upper yield strength is described by the theory of delay of yield of Clark, Cottrell, and Yu. N. Rabotnov.

In contrast to metals for the majority of geological materials the $\tau-\gamma$ curve depends significantly upon the amount of compression p. Its typical form is shown in Fig. 5d. (The value of $\gamma$ is understood phenomenologically as the ratio of the mutual displacement of the linkages to the layer thickness (Fig. 5b).)

According to the experimental investigations of Byerlee [5] for a large number of various rocks at fracture the following simple relationship occurs:

$$
\tau_{\mathrm{u}}=\left\{\begin{array}{l}
0.85\left(p-p_{0}\right) \text { with } 0<p-p_{0}<200 \mathrm{MPa} \\
50 \mathrm{MPa}+0.6\left(p-p_{0}\right) \text { with } p-p_{0}>200 \mathrm{MPa}
\end{array}\right.
$$

where po is the interstitial pressure of the liquid impregnating the rock.

From the preceding sections 2 and 3 it follows that:

a) plastic supercritical deformations propagate under dynamic conditions along some surface in the form of a slip crack (dislocation rupture);

b) the falling branch of the $T-\gamma$ curve (including the value $\gamma_{*}-\gamma_{u}$ ) is a functional dependent upon the geometry of the specimen, the rigidity of fastening, etc.;

c) the value of $\tau_{s}$ is the specific force of friction between the edges of a slip crack.

These theoretical conclusions agree well with experience and with modern concepts on earthquakes $[1,6,7]$. Let us recall that according to these concepts an earthquake occurs as the result of propagation of a dynamic slip crack along some site with an area of $d^{2}$ and the seismic energy 


$$
E_{e}=\frac{\left(\tau_{\mathrm{u}}-\tau_{s}\right)^{2}}{2 G} d^{3}
$$

is liberated where $G$ is the shear modulus.

The basic reasons for earthquakes are local stress concentration as the result of creep of the filler in fractures [8,9], movement of the substance of the mantle causing a change in the tectonic stresses in the solid crust of the Earth, filtration of water or steam into the zone of the origin, etc. The presence of a seismogeneous zone in the crust of the earth at a depth of approximately 3 to $15 \mathrm{~km}$ is explained first by an increase in the difference $\tau_{u}-\tau_{s}$ for rocks with an increase in depth [10].

5. Conclusion. The basic conclusion of the work may be formulated in the following manner. Supercritical deformations of solids within the limits of the theory of infinitely small deformations are always concentrated in infinitely narrow zones simulated by surfaces of discontinuity of the displacements.

The author thanks G. I. Bykovtsev and L. Ya. Galushko for discussion of the work and helpful remarks.

\section{LITERATURE CITED}

1. G. P. Cherepanov, Mechanics of Brittle Fracture, McGraw-Hill, New York (1979).

2. G. P. Cherepanov, "The problem of nonuniqueness in the theory of plasticity," Dokl. Akad. Nauk SSSR, 218, No. 4, 1124-1127 (1974).

3. J. Taylor, "The interfaces between viscous liquids in narrow crevices," in: Problems of the Mechanics of a Continuum [in Russian], Izd. Akad. Nauk SSSR, Moscow (1961), pp. $720-726$.

4. G. P. Cherepanov, "Point defects in solids," in: Eshelby Memorial Volume: Fundamentals of Deformation and Fracture, Cambridge Univ. Press (1984).

5. J. Byerlee, "Brittle-ductile transition in rocks," J. Geophys. Res., 73, 4741-4750 (1968).

6. B. V. Kostrov, The Mechanics of Origin of a Tectonic Earthquake [in Russian], Nauka, Moscow (1975).

7. K. Ak1 and P. G. Richards, Quantitative Seismology, W. H. Freeman, San Francisco (1980).

8. G. P. Cherepanov, "The development of cracks under creep conditions," in: Nonlinear Problems of the Mechanics of a Solid [in Russian], Nauka, Moscow (1984), pp. 221-235.

9. G. P. Cherepanov, "One mechanism of the development of fractures in the solid crust of the earth," Izv. Akad. Nauk SSSR, Fiz. Zemli, No. 9, 3-12 (1984).

10. J. R. Rice, Constitutive Relations for Fault Slip and Earthquake Instabilities (1983). (Preprint, Harvard Univ. Press). 